\title{
On the origin of solar and stellar flares
}

\author{
Subhon Ibadov ${ }^{1,2}$ and Firuz S. Ibodov ${ }^{1}$ \\ ${ }^{1}$ Moscow State University, Sternberg Astronomical Institute \\ 119991 Moscow, Russia \\ email: mshtf@sai.msu.ru \\ ${ }^{2}$ Institute of Astrophysics, Tajik Academy of Sciences, \\ 734042 Dushanbe, Tajikistan \\ email: ibadovsu@yandex.ru
}

\begin{abstract}
Solar and stellar flares due to impacts of comet nuclei and falling evaporating bodies, FEBs, with the Sun/stars are analytically considered. It is shown that impacts of sun/stargrazing comets will be accompanied by essential aerodynamic effects: nuclei crushing and expansion/ flattening of crushed mass within the chromosphere. These processes lead to impulse generation of a hot plasma, strong shock wave in the thin layer near photosphere, eruption of the hot ionized clump to the lower corona, i.e., impact-induced solar/stellar flares.
\end{abstract}

Keywords. Sun/stargrazing comets, falling evaporating bodies/FEBs, impacts, stellar flares

\section{Introduction}

Data from solar space missions (SOLWIND, SMM, SOHO, STEREO, SDO) and ground-based observations as well as spectral observations of young stars like Beta Pictoris together with celestial mechanics calculations indicate the presence of sun/stargrazing comet/FEB fluxes passing close to the solar/stellar surface, at distances less than $0.01 \mathrm{AU}$ (e.g., Marsden 1989, Bailey et al. 1992, Beust et al. 1996, http://sungrazer.nrl.navy.mil/). Besides, calculations show that solar photospheric thermal radiation leads to a very limited decrease in the cometary nuclei radii, less than 10-20 m, in the 'classical'/vacuum approach (e.g. Weissman 1983, MacQueen \& St.Cyr 1991).

Meantime, parabolic velocity of comets near the solar surface is $V_{o}=617 \mathrm{~km} / \mathrm{s}$, while the density of the solar atmosphere within the chromosphere considerably exceeds $10^{-15} \mathrm{~g} / \mathrm{cm}^{3}$ being $10^{-8}-5 \times 10^{-7} \mathrm{~g} / \mathrm{cm}^{3}$ in the photosphere, so that aerodynamic pressure on the comet nuclei will considerably exceed the tension for mechanical disintegration. At the same time the specific kinetic energy of comet nuclei in the inner heliosphere, especially near the surface of the Sun, more than thousand times exceeds the evaporation/sublimation energy of comet nuclei material, i.e., of the order of $10^{10}$ $\mathrm{erg} / \mathrm{g}$. Hence, high-temperature explosive phenomena due to comet/FEBs impacts with the Sun/stars are possible and should be considered as one of possible processes giving rise to solar/stellar flares: there are well-known planetary analogs as the 1908 Tunguska and 2013 Chelyabinsk air explosions ( Grigorian 1980, Ibadov et al. 2008, Ibadov et al. 2009, Grigorian et al. 2013 and references therein).

We present completely analytic approach in investigating the passage of comet nuclei/FEBs through the stars atmosphere for revealing peculiarities of an impact mechanism of solar/stellar flares. 


\section{Aerodynamic heating and crushing of comet nuclei/FEBs in solar/stellar atmospheres}

For a comet approaching the Sun the critical density of the solar atmosphere at which the energy flux on the surface of the nucleus due to collisions with atoms of the solar atmosphere becomes equal to that due to irradiation by the solar photosphere thermal emission will be $\rho_{c r}=L_{o} /\left(2 \pi R_{o}^{2} V^{3}=5 \times 10^{-13} \mathrm{~g} / \mathrm{cm}^{3}\right.$ with $L_{o}$ and $R_{o}$ as the solar luminosity and radius, respectively. The numerical value obtained corresponds to the density of the solar atmosphere near the upper boundary of the chromosphere.

A criterion for the onset of a comet nucleus aerodynamic crushing $P_{a^{*}}=\rho_{a^{*}} V_{*}^{2}=$ $\sigma_{*}$ together with the equation for aerodynamic deceleration of a constant-mass comet nucleus, i.e., that having sufficiently large initial radius, $R_{n}>100 \mathrm{~m}$, written as

$$
M \frac{d V}{d t}=\frac{1}{2} C_{x} S \rho_{a} V^{2}
$$

allow us to find the onset height for the nucleus aerodynamic destruction, namely

$$
h_{*}=H \ln \left(\frac{\rho_{o} V_{o}^{2}}{\sigma}\right) \text {. }
$$

Here $M, C_{x}$ and $S$ are the mass, drag coefficient and cross-section of a comet nucleus; $\rho_{a}(h)=\rho_{o} \exp (-h / H)$ is the density distribution of atmosphere; $h$ is the height for which the limb of the Sun's disk with the optical density $\tau_{\lambda}(\lambda=5000 \AA)=0.005$ is taken as the reference point and $\rho_{o}=\rho_{a}(h=0)=10^{-8} \mathrm{~g} / \mathrm{cm}^{3} ; \mathrm{H}$ is the local height scale: $H=3 \times 10^{3} \mathrm{~km}$ and $200 \mathrm{~km}$ for the chromosphere and photosphere, respectively; $V, V_{o}$ are the nucleus velocity in the atmosphere and its initial velocity; $P_{a^{*}}, \rho_{a^{*}}, V_{*}$ are the frontal pressure of the incoming atmospheric gas, mass density of the atmosphere, comet nucleus velocity - all these three values correspond to the onset of the nucleus aerodynamic crushing, $\sigma_{*}$ is the strength of the nucleus material.

Substituting into $(2.2) H=3 \times 10^{8} \mathrm{~cm}, \rho_{o}=10^{-8} \mathrm{~g} / \mathrm{cm}^{3}, \sigma_{*}=10^{4} \mathrm{dyn} / \mathrm{cm}^{2}$ we obtain the crush-height in atmosphere $h_{*}=10^{9} \mathrm{~cm}$. The corresponding characteristic atmosphere density will be $\rho_{a^{*}}=\sigma_{*} / V_{o}^{2}=3 \times 10^{-12} \mathrm{~g} / \mathrm{cm}^{3}$. Accordingly, the characteristic intensity of energy flux onto the nucleus due to aerodynamic heating will be $\left(\rho_{a} * V_{o}^{3}\right) / 2=3 \times 10^{11} \mathrm{erg} /\left(\mathrm{cm}^{2} \mathrm{~s}\right)$ : it is four times more than the maximum energy flux from the solar thermal radiation.

It should be noted that according to the solution of the deceleration Eq. (2.1) the velocity of large bodies above the solar photosphere will be practically constant, $V=V_{o}$.

So, the above analytic data indicate that within the solar chromosphere an intense aerodynamic crushing of comet nuclei occurs. Besides, the intensity of energy flux due to aerodynamic heating will be strongly more that by solar thermal radiation, i.e., the comet nuclei disintegration phenomenon near the Sun/stars acquires completely meteoric character (cf. Grigorian et al. 1997, Ibadov et al. 1999, Ibadov et al. 2007).

\section{Aerodynamic explosion of FEBs in atmosphere: photospheric solar/stellar flares}

The rapid rise of aerodynamic pressure at the entry of the nucleus into denser atmosphere layers leads to the situation when the destruction being started locally propagates through the nucleus body from the frontal surface to the rear one. At the same time the crushed mass loses its integrity, spreading in the lateral direction under the action of pressure gradient at the frontal surface. So, to take into account these aerodynamic effects we have to modify basic equations of the physical theory of meteors, like made 
for the 1908 Tunguska explosion of the fireball/superbolide origin. Then the equation for aerodynamic deceleration, Eq.(1), for the completely fragmented transversally expanding nucleus/meteoroid in the constant-mass approximation, that is acceptable due to essential decrease of the coefficient for heat transfer at high temperatures for high-velocity large meteor bodies because of shielding effects (cf. Grigorian 1980), will be reduced to the following integral equation:

$$
\int_{\tilde{V}}^{V} \frac{d V}{V}=-\frac{3 C_{x} b \rho_{o} H}{8 R_{n}^{3} \nu \rho_{n} \sin \alpha} \int_{\tilde{r}}^{r} R^{2} d r .
$$

Here $R=R[r(h)]$ is the law for increase of the transverse radius of the flattening crushed mass:

$$
\frac{R}{2 R_{n}}=1+\frac{\sqrt{b / 2}}{C}(\sqrt{1+r}-\sqrt{1+\tilde{r}})
$$

$R_{n}, \rho_{n}$ are the initial radius and the density of the comet nucleus, respectively; $\alpha$ is the angle between the entry velocity of the nucleus into the atmosphere and the local horizon;

$$
\begin{gathered}
b=\nu \exp \left(-\frac{h_{*}}{H}\right) ; \quad \nu=\frac{3 C_{x} \rho_{o} H}{4 \rho_{n} R_{n} \sin \alpha} ; \\
C=\left(\frac{3 C_{x} R_{n} \sin \alpha}{8 H}\right)^{1 / 2} ; \quad r=\left[\exp \left(\frac{h_{*}-h}{H}\right)\right]-1 ; \quad \tilde{r} \approx \frac{4 C^{2}}{b} ;
\end{gathered}
$$

furthermore, $\nu$ is the parameter of the aerodynamic deceleration, it is very small for large bodies; $\tilde{r}, \tilde{h}, \tilde{V}$ are the characteristic values of $\mathrm{r}, \mathrm{h}$ and $\mathrm{V}$, which correspond to the value of $R=2 R_{n}$, i.e., to the time instant when the nucleus is completely crushed and its transverse radius is equal to the doubled value of the initial radius.

An analytic solution of Eq. (3.1), using Eq. (3.2), for the region close to the endpoint of the deceleration trajectory in atmosphere, i.e., at small distances from the solar/stellar surface, $h \ll \tilde{h} \ll h_{*}, r>>1$, gives the law for velocity variation of completely crushed FEBs near the stellar photosphere in the form:

$$
V=\tilde{V} \exp \left[-\frac{b^{2}}{2 C^{2}}\left(r^{2}-\tilde{r}^{2}\right)\right]=V_{o} \exp \left(-\frac{b^{2}}{2 C^{2}} r^{2}\right) .
$$

From Eqs. (3.4), (3.5) we can find the height-range of the basic deceleration of the $\mathrm{FEB}$, where the decrease of velocity from $V_{1}=0.9 V_{o}$ to $V_{2}=0.1 V_{o}$ occurs, namely

$$
\Delta h_{d}=h_{2}-h_{1}=H \ln \frac{1+r_{1}}{1+r_{2}}=H \ln \frac{r_{1}}{r_{2}} \approx 0.7 H .
$$

Using Eqs. (2.1), (3.2), (3.4), (3.5) an equation for the rate of energy loose by the FEB in atmosphere may be obtained, in the form of $d E / d r$ as a function of $r=r(h)$. On the basis of this equation explicit analytical expressions may be found for the parameters of the site where the maximum energy release due to aerodynamic deceleration of the crushed and flattening FEB, "explosion", in atmosphere takes place (cf. Grigorian et al. 2013):

$$
\begin{gathered}
r_{m}=r_{e x}=\frac{C}{b} \\
h_{m}=h_{e x}=h_{*}-H \ln \left(1+\frac{C}{b}\right)=H \ln \left[\frac{\rho_{o} V_{o}^{2}}{(1+C / b) \sigma_{*}}\right],
\end{gathered}
$$




$$
\begin{gathered}
R_{m}=R_{e x}=2 R_{n}\left(1+\frac{1}{\sqrt{2 C}}\right), \\
V_{m}=V_{e x}=\frac{V_{o}}{\sqrt{e}} .
\end{gathered}
$$

Accepting $C_{x}=1, \rho_{n}=0.5 \mathrm{~g} / \mathrm{cm}^{3}, R_{n}=10^{5} \mathrm{~cm}, \sin \alpha=0.5, \sigma_{*}=10^{6} \mathrm{dyn} / \mathrm{cm}^{2}$ from (3.3), (3.4), (3.7), (3.8), (3.9) we have $\nu=7 \times 10^{-5}, C=7 \times 10^{-2}, b=3.5 \times 10^{-6}$, $r_{e x}=2 \times 10^{4}, h_{e x}=-7 H=-1400 \mathrm{~km}, R_{e x}=7.4 R_{n}=7.4 \mathrm{~km}$.

The explosive energy deposited in the zone of maximum deceleration, $E_{m}=E_{e x}$, is determined by the initial mass of the comet nucleus, $M_{o}$, coming into the atmosphere, and the mass of the atmosphere within the decelerating layer, $\Delta M_{a}$. Usage of $\rho_{a}(h)$ and Eq.(3.6) gives $\Delta M_{a} \approx M_{o}$, so that with the help of Eq. (3.10) an analytic relation to estimate the energy of "explosion" may be found as:

$$
E_{e x}=\frac{M_{o} \Delta M_{a} V_{e x}^{2}}{2\left(M_{o}+\Delta M_{a}\right)}=\frac{\rho_{n} R_{n}^{3} V_{o}^{2}}{3 e} .
$$

On the basis of Eqs. (3.6) and (3.10) the characteristic time for thermalization of the kinetic energy of the fragmented nucleus may be estimated:

$$
\tau_{e x}=\frac{\Delta h_{d}}{V_{e x}}=\frac{0.7 \sqrt{e} H}{V_{o}} \approx \frac{H}{V_{o}}=0.3 \mathrm{~s},
$$

that explicitly indicates an impulsive and strongly explosive character of the energy release process in the near photosphere decelerating layer.

According to Eq. (3.11) the explosive energy will be around the energy of large solar flares, $10^{32} \mathrm{erg}$, for impacts of comets like comet Halley 1986 III while FEBs like comet Hale-Bopp 1995 OI can lead to superflares (cf. Grigorian et al. 2000, Ibadov et al. 2009, Eichler \& Mordecai 2012).

The asymptotic velocity of expansion of a hot cylindrical plasma column in the near photosphere explosive layer, i.e., the initial velocity of a "blast" shock wave that will provide eruption of a hot plasma clump/plume from the solar/star surface may be determined using the analogy with the expansion of a short-living hot plasma clots, "compound particles", produced by collision between two high-velocity dust particles (see, e.g., Ibadov 1990):

$$
V_{s h}=\left[\frac{k T_{o}}{2 \pi A m_{p}}+\frac{3 k(1+z) T_{o}}{A m_{p}}\right]^{1 / 2},
$$

where $T_{o}$ is the initial temperature of plasma in the explosive layer:

$$
T_{o}=\frac{A m_{p} V_{e x}^{2}}{12 k\left[\left(1+z+\left(2 x_{1}\right) / 3\right)\right]},
$$

$A$ is the mean atomic number for FEB material and matter of photosphere, $m_{p}$ is the proton mass, $k$ is the Boltzmann constant, $z$ is the mean multiplicity of ions charge, $x_{1}$ is the mean relative ionization potential (Ibadov 1986, 1996, 2011).

The maximum height of the photospheric mass ejections due to cometary impacts may be estimated as:

$$
h_{m}=\frac{V_{s h}^{2}}{2 g_{o}}=\frac{R_{o}^{2} V_{s h}^{2}}{2 G M_{o}} .
$$

Here $g_{o}$ is the gravity acceleration on the star's surface, $G$ is the gravity constant, $M_{o}$ is the mass of the Sun/star. 
It should be noted that "blast" wave generated in the near-photosphere decelerating layer can be considered as a "strong" shock wave. Indeed, the initial pressure in the front of shock wave generated due to explosive energy release in the layer, according to (3.6), (3.9), (3.11) will be

$$
P_{s h}=\frac{E_{e x}}{\pi \Delta h_{d} R_{e x}^{2}}=3 \times 10^{5} \rho_{n} R_{n} \approx 10^{10} \mathrm{dyn} / \mathrm{cm}^{2}=10^{4} \mathrm{~atm},
$$

that is greater than the ambient photospheric pressure more than $10^{4}$ times, for $R_{n} \geqslant$ $10^{5} \mathrm{~cm}$.

Using (3.13)-(3.15) with realistic values of $A=20, z=5, x_{1}=3, M_{o}=2 \times 10^{33} \mathrm{~g}$ we get $T_{o}=7 \times 10^{6} \mathrm{~K}, V_{s h}=1.7 \times 10^{7} \mathrm{~cm} / \mathrm{s}, h_{m}=5 \times 10^{9} \mathrm{~cm}$.

It is known that there are variety of solar prominences having maximum heights in the range 30-50 thousand kilometers (e.g. Filippov et al. 2006, Harrison et al. 2010). Hence, we can note that FEBs impact generated photospheric mass ejections can form a certain type of solar/stellar prominences having relatively high abundance of metal atoms ( Ibodov \& Ibadov 2011, Ibodov \& Ibadov 2014).

The analytic approach presented had been tested explaining the HST data obtained during observations of ejecta from collision of comet SL 9 with Jupiter in 1994 ( Hammel et al. 1995) and also studying the 2013 Chelyabinsk event (Grigorian et al. 2013 and references therein).

\section{Conclusions}

Impacts of comet nuclei/FEBs with the Sun/stars will lead to impulse generation of a hot plasma, "explosion", strong "blast" wave, ejection of a hot plasma plume to the heights reaching the lover corona.

Impact mechanism of solar flares is capable to lead to solar/stellar superflares: it is reasonable coordinated observations/monitoring of short perihelion comets and nearby young stars abundant in FEBs.

The study of comet impact-generated high-temperature plasma phenomena in the solar photosphere/chromosphere by space telescopes like SDO, having high spatio-temporal resolutions, are of interest for the physics of solar/stellar flare activity as well as physics of comets.

\section{Acknowledgements}

The authors are grateful to SOC/LOC, 29th IAU General Assembly, for invitation (Honolulu, Hawaii, USA, 3-14 August 2015), SAI MSU for hospitality, Dr. G.M.Rudnitskij for useful discussions, Drs.E.Shimanovskaya and N.Gostev for technical support, as well as D. Talbott and S. Schirott for the support of the trip.

\section{References}

Bailey, M. E. \& Chambers, J. E., Hahn G. 1992, A\& A, 257, 315

Beust, H., Lagrange, A.-M., Plazy, F., \& Mouillet, D. 1996, A\&A A, 310, 181

COSPAR 1998, COSPAR Inform. Bull., 142, 22

Eichler, D. \& Mordecai, D. 2012, ApJL, 761, L27

Filippov, B. P., Zagnetko, A. M., \& Ajabshirizadeh, A., Den O. G. 2006, Sol. Sys. Res., 40, 319

Grigorian, S. S. 1980, Cosmic Res., 17, 724

Grigorian, S. S., Ibodov, F. S., \& Ibadov, S. 1997, Phys.-Dokl., 42, 262 
Grigorian, S. S., Ibadov, S., \& Ibodov, F. S. 2000, Dokl.-Phys., 45, 463

Grigorian, S. S., Ibodov, F. S., \& Ibadov, S. 2013, Sol. Sys. Res., 47, 268

Hammel, H. B., Beebe, R. F., \& Ingersoll, A. P., et al. 1995, Science, 267, 1288

Harrison, R. A., Davis, C. J., \& Bewsher, D., et al. 2010, ASR, 45, 1

Ibadov, S. 1986, ESA SP-250, 1, 377

Ibadov, S. 1990, Icarus, 86, 283

Ibadov, S. 1996, Physical Processes in Comets CI, M., 181

Ibadov, S. 2011, in: A. Manchado, L.Stanghellini, \& D. Schonberner (eds.), Proc. IAUS 283, CUP, p.392

Ibadov, S. \& Ibodov, F. S. 2015, in: G. Meynet, C. Georgy, J. Groh \& P. Stee (eds.), Proc. IAUS 307, p., 108

Ibadov, S., Ibodov, F. S., \& Grigorian, S. S. 2007, in: J. Bouvier \& I. Appenzeller (eds.), Proc. IAUS 243, P.VI.4

Ibadov, S., Ibodov, F. S., \& Grigorian, S. S. 2008, in: V. Svettsov (ed.), Proc. Intl. Conf. "100 Years Tunguska Phenomenon", RAS, M., p.45

Ibadov, S, Ibodov, F. S. \& Grigorian, S. S. 2009, in: N.Gopalswamy \& D.F. Webb (eds.), Proc. IAUS 257, CUP, p.341

Ibodov, F. S. \& Ibadov, S. 2011, in: A.Bonanno \& A. Kosovichev (eds.), Proc. IAUS 274, CUP, p.92

Ibodov, F. S. \& Ibadov, S. 2014, in: B. Schmieder, J.-M. Malherbe, \& S.T. Wu, (eds.), Proc. IAUS 300, CUP, p. 509

\&MacQueen, R. M., St.Cyr, O. C. 1991, Icarus, 90, 96

Marsden, B. G. 1989, AJ, 98, 2306

Weissman, P. R. 1983, Icarus, 55, 448 